ISSN 1112-9867

http://www.jfas.info

\title{
PREDICTION OF RAINFALL BASED ON WEATHER PARAMETER USING ARTIFICIAL NEURAL NETWORK
}

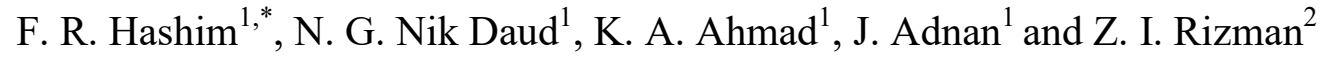 \\ ${ }^{1}$ Department of Electrical and Electronic Engineering, Faculty of Engineering, National \\ Defense University of Malaysia, Sungei Besi Camp, 57000 Kuala Lumpur, Malaysia \\ ${ }^{2}$ Faculty of Electrical Engineering, UniversitiTeknologi MARA, 23000 Dungun, Terengganu, \\ Malaysia
}

Published online: 10 September 2017

\begin{abstract}
Artificial neural networks which are inspired by the concept of the biological neurons are commonly used in many applications including in the field of weather forecasting. The neural networks approaches have provided an educated solution to aid in the decision-making process for weather forecasting as well as a viable means of the prediction of raindrop. This paper attempts to determine the suitability and the applicability of artificial neural networks for rain prediction based on temperature, pressure and humidity. Those conditions have been used as input data and solution was classified as percentage of raining. Multilayer perceptron network with two different learning algorithms have been studied. The multilayered perceptron trained using Lavenberg Marquardt algorithm has been proven to produce better results with accuracy percentage (99.75\%) as compared to back propagation $(94.57 \%)$.
\end{abstract}

Keywords: multilayer perceptron; rainfall prediction; temperature; pressure; humidity.

Author Correspondence, e-mail: fakroul@upnm.edu.my

doi: http://dx.doi.org/10.4314/jfas.v9i3s.38 


\section{INTRODUCTION}

A neural network can be seen as a machine that is designed to model the way in which the brain performs a particular task or function of interest [1], where it processes information in a similar way the human brain does. Neural network has successfully been applied in various fields including financial, engineering and mathematics by offering useful properties and capabilities, i.e. nonlinearity, input-output mapping and neurobiological analogy. In those fields, neural networks have been configured to identify systems, as pattern recognition system, in digital image processor as well as data classification techniques with significant success rate.

Weather conditions give a big impact in developing certain population. Development population is measured by the surrounding phenomena such as rain tabulation, temperature reading and the location itself. Weather condition predictions can be made through a deep analysis on temperature, humidity and air pressure. These three elements might the occurrence of mother-nature phenomena such as heavy rain, big hurricane and huge cyclone. For example, the movement of the air from high pressure area to low pressure area will produce the hurricane or cyclone based on the difference of pressure reading. The hurricane or cyclone become greater since the difference of air pressure is huge[2-6].

The combination of temperature, pressure and humidity can be the indicator for prediction of raindrops. Since the temperature is very high so the percentage of raindrop will increase. High reading from the humidity sensor also give a high percentage of the raindrop to occur. Humidity condition will produce a big cloud compared to desert area with low reading of humidity and only produces tiny clouds. Air pressure transformations to low pressure location form high pressure location also inviting the raindrop, since the wind will blow from the high pressure location to the low pressure location. The complete combination of high temperature reading, high humidity reading and low pressure reading give the high percentage of raindrop to occur.

To compose the raindrop prediction, the historical data was collected to train the neural network system by analyzing the pattern of raindrop model. Since the recognition of raindrop model was done, the neural network system will be test in term to confirm either the model of 
raindrop is suitable to analyze by neural network system and capable to give the high accuracy or not. The neural network system was proven suitable to predict the raindrop and capable to give a high accuracy, the real time data is needed to predict real time condition. Since the neural network system capable to give the high accuracy thus the raindrop condition will be predicted accurately.

\section{MULTILAYER PERCEPTRON NETWORK}

Artificial Neural Network (ANN) is an information processing paradigm that is inspired by the concept of biological neural cells which are known as neurons, process information. Neurons are the tiny cells that our brains are composed of to detect the outside environment and body's internal environment, to formulate behavioral responses to those signals and to control bodies based on the chosen responses. A biological neuron is defined as a collection of about 10 billion interconnected neurons. Biological neurons are the naturally occurring equivalent of the artificial neural network. Artificial neural networks are proposed based on the concept that they are capable to model biological neurons structures in terms of both their architecture and operation. They function as mathematical computational models for non-linear function approximation, data classification, and clustering/non-parametric regression. Artificial neural networks are capable in placing of the human brain in providing performance reliability especially in decision-making. Hence, that can act as an alternative to intelligent systems with the used of Multilayered Perceptron (MLP) network as one of the commonly used artificial neural networks. 


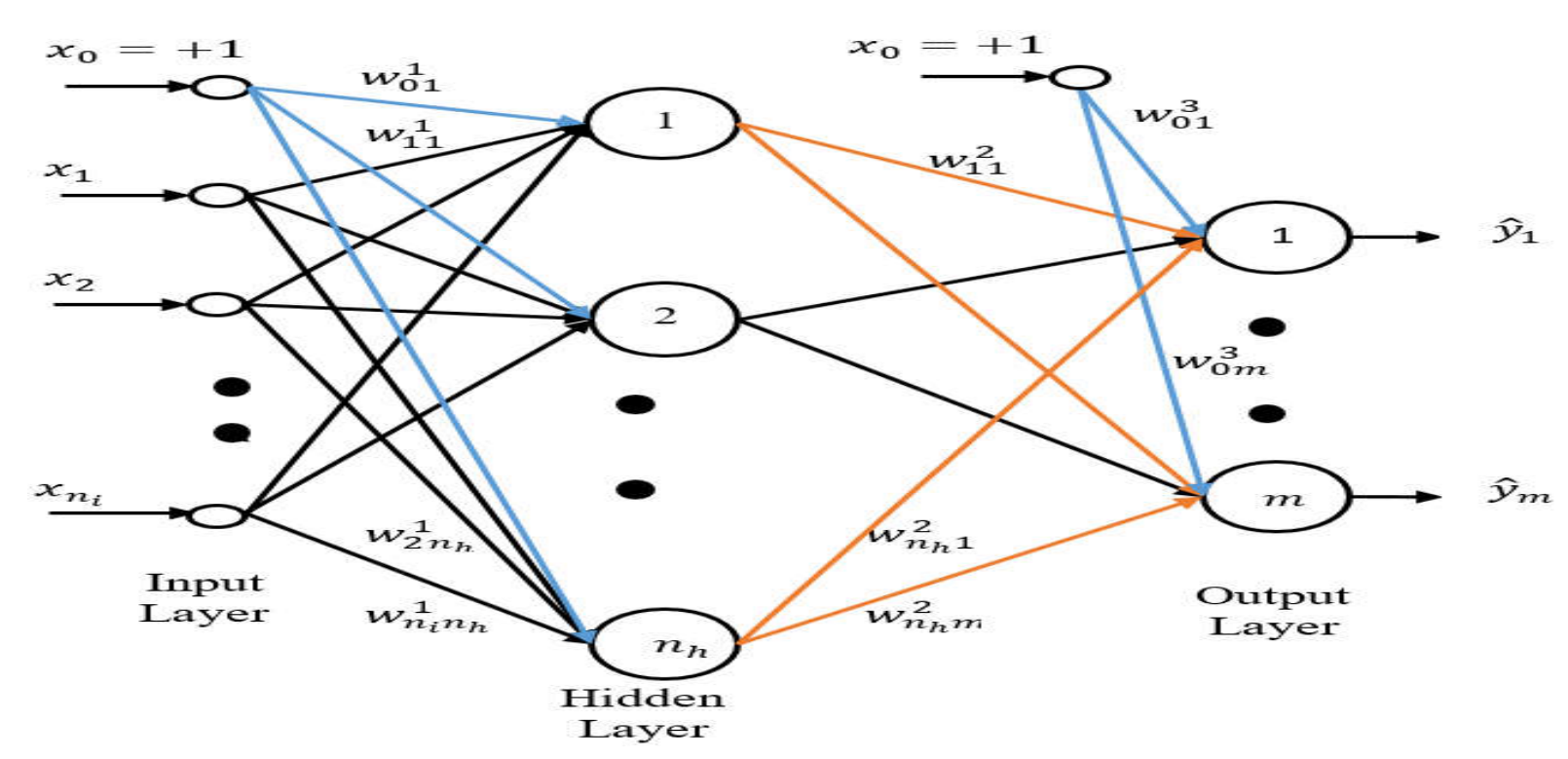

Fig.1. Architecture of the MLP network

In 1958, Rosenblatt introduced his perceptron brain model that shows the neural network first appeared in learning procedure [15]. Cascading of several perceptron models in a layer produce a network as shown in Fig. 1, which represent a generalization of the single-layered perceptron. The structure in Fig. 1 shows a MLP network with $m$ and $n_{i}$ as the number of output nodes and input nodes respectively, and $n_{h}$ as the number of hidden nodes can be expressed by following equation:

$\hat{y}(t)=\sum_{j=1}^{n_{h}} w_{j k}^{2} F\left(\sum_{i=1}^{1} w_{i j}^{1} x_{i}^{0}(t)+b_{j}^{1}\right)$ for $1 \leq k \leq m$

where $w_{i j}^{1}$ and $w_{j k}^{2}$ denote the weights of the connection between input and hidden layer, and weights of the connection between hidden and output layer respectively. $b_{j}^{1}$ and $x_{i}^{0}$ denote the thresholds in hidden nodes and inputs that are supplied to the input layer respectively. $F($.$) is an activation function and is normally being selected as sigmoid function.$

From Equation (1), the values of $w_{i j}^{1}, w_{j k}^{2}$ and $b_{j}^{1}$ areunknown and should be determined using appropriate algorithm. In this paper, Back Propagation (BP) algorithm is proposed to estimate the weights as it is commonly used to find optimum values for those parameters. This algorithm is based on the error-correction learning rule. This paper will also employ another learning algorithm to train the MLP network, which is Levenberg Marquardt and the performance of these training algorithms will be compared later. 


\subsection{Back Propagation Algorithm}

As the most commonly used training algorithm, Back-Propagation is a gradient descent procedure that computes the derivatives' values in an efficient way, and modifies the weights according to a parameter known as the learning rate [16]. Back propagation is the steepest decent type algorithm where the weight connection between the i-th neuron of the input layer and the $\mathrm{j}$-th neuron of the hidden layer are respectively updated according to:

$$
\begin{gathered}
w_{j i}(t)=w_{j i}(t-1)+\Delta w_{j i}(t) \\
b_{j}(t)=b_{j}(t)+\Delta b_{j}(t)
\end{gathered}
$$

The increment $\Delta w_{j i}(t)$ and $\Delta b_{j}(t)$ given by:

$$
\begin{aligned}
\Delta w_{j i}(t) & =\eta_{w} \rho_{j}(t) x_{i}(t)+\alpha_{w} \Delta w_{j i}(t) \\
\Delta b_{j}(t) & =\eta_{b} \rho_{j}(t) x_{i}(t)+\alpha_{w} \Delta b_{j}(t)
\end{aligned}
$$

where the subscripts $w$ and $b$ represent the weight and threshold respectively. The momentum constants, $\alpha w$ and $\alpha b$ which determine the influence of the past parameter changes on the current direction of movement in the parameter space, $\eta_{w}$ and $\eta_{b}$ represent the learning rates and $\rho_{j}(t)$ is the error signal of the $j$-th neuron of the hidden layer which is back propagated in the network. The error signal at the output node is

$\rho(t)=y_{k}(t)-\hat{y}_{k}(t)$

where $y_{k}(t)$ is the expected output, since the activation function of the output neuron is linear. For the neurons in the hidden layer

$\rho_{j}(t)=F^{\prime \prime}\left(x_{i}(t)\right) \sum_{j} \rho_{j}^{k}(t) w_{j k}^{2}(t-1)$

where $F^{\prime \prime}\left(x_{i}(t)\right)$ is the first derivative of $F\left(x_{i}(t)\right)$ with respect to $x_{i}(t)$. The algorithm suffers from a slow convergence rate since back propagation algorithm is the steepest decent type algorithm. The algorithm can be sensitive to the user selectable parameters and the search for the global minima may become trapped at local minima. [17].

\subsection{Levenberg-Marquardt Algorithm}

The Levenberg-Marquardt algorithm is a gradient-based, deterministic local optimization algorithm. The advantage of the Levenberg-Marquardt over the traditional Back Propagation algorithm once employed to train the MLP model is that it can provide a faster (second-order) 
convergence rate and keep relative stability [18-19].

The Levenberg-Marquardt algorithm was designed to approach second-order training speed without having to compute the Hessian matrix like the quasi-Newton methods. The Hessian matrix can be approximated as expressed in Equation (6) later when the performance function has the form of a sum of squares (as is typical in training feed forward networks):

$H=J^{T} J$

and the gradient can be computed as:

$g=J^{T} \rho$

where $J$ is the Jacobian matrix that contains first derivatives of the network errors with respect to the weights and biases, and e is a vector of network errors. Through a standard back propagation technique, the Jacobian matrix can be computed, which is much less complex than computing the Hessian matrix [20]. The Levenberg-Marquardt algorithm uses this approximation to the Hessian matrix in the following Newton-like update:

$\Delta w=-\left[J^{T} J+\mu I\right]^{-1} J^{T} \rho$

where $\Delta w$ is a differential weights and $\mu$ is a control parameter. When the scalar $\mu$ is zero, this is just Newton's method, using the approximate Hessian matrix. When $\mu$ is large, this becomes gradient descent with a small step size. Newton's method is faster and more accurate near an error minimum, so the aim is to shift towards Newton's method as quickly as possible. Thus, $\mu$ is decreased after each successful step or which also known as reduction in performance function and is increased only when a tentative step increases the performance function. In this way, the performance function will always be reduced in each iteration of the algorithm [20].

\section{METHODOLOGY}

Current method of raindrop prediction was using an expensive gadget. This gadget operated along with temperature, pressure, humidity and other parameters in term of raindrop prediction. In related study, in [13] has showed temperature, pressure and humidity itself can be the only parameters to predict the raindrop. The study reported the raindrop occur by combination of the reading from temperature sensor, pressure sensor and humidity sensor. The 
combination reading of high temperature, low pressure and high humidity will trigger the raindrop alarm. The samples consist of 5327 numbers of data with 3000 data are used to train the MLP networks, while the other 2327 data are used to test the MLP networks. Both training and testing phase show the suitability and capability of MLP networks to make the right prediction of the raindrop.

\section{RESULTS AND DISCUSSION}

Similar to the procedure used by [22-23] for data classification, this study applies two analyses. Firstly, optimum structure analysis implemented to determine the optimum number of hidden nodes that can produce the highest river's water quality classification. For this analysis, the MLP network is trained with 10000 epochs for the MLP network trained using BP and LM algorithms respectively. Table 1 shows the result of the analysis for different types of training algorithm.

Table 1.Results for the optimum structure analysis of the MLP network trained using different training algorithms

\begin{tabular}{ccc}
\hline $\begin{array}{c}\text { MLP Network } \\
\text { Training Algorithm }\end{array}$ & $\begin{array}{c}\text { Optimum Number } \\
\text { of Epochs }\end{array}$ & $\begin{array}{c}\text { Optimum Number } \\
\text { of Hidden Nodes }\end{array}$ \\
\hline BP & 36 & 9 \\
LM & 5 & 3 \\
\hline
\end{tabular}

The second analysis that is the performance comparison analysis then applied using the optimum structure of the MLP network obtained in the first analysis. The analysis is done based on the accuracy of correct prediction of raindrop. Table 2 shows the result of the analysis for training and testing phase.

Table 2. Results for the performance analysis of the MLP network trained using different training algorithms

\begin{tabular}{cccc}
\hline $\begin{array}{c}\text { MLP Network Trained Using Different } \\
\text { Training Algorithm }\end{array}$ & Training (\%) & Testing (\%) & Overall (\%) \\
\hline BP & 95.67 & 93.47 & 94.57 \\
LM & 99.90 & 99.60 & 99.75 \\
\hline
\end{tabular}


The results tabulated in Table 1 show that the MLP network trained using the Lavenberg Marquardt (LM) form simpler network architecture, as it only requires 5 epochs and 3 hidden nodes than back propagation (BP) training algorithms requires 36 epochs and 9 hidden nodes. For the performance analysis, the results obtained as tabulated in Table 2 show that the MLP network trained using LM algorithm produces better performance of accuracy than BP with $99.90 \%$ and $99.60 \%$ for training and testing phase respectively, which gives overall accuracy of $99.75 \%$. This shows that the MLP network trained using LM has lower convergence rate as compared to BP.

\section{CONCLUSION}

Temperature, pressure and humidity parameters are used as the indicator to predict the raindrop. A lot of conventional methods are used in terms of to raindrop prediction. In this study, neural network method is used to analyze the raindrop prediction. The results show that artificial neural network is suitable and capable to predict the raindrop based on temperature, pressure and humidity reading. Further improvement can be made to upgrade the performance of the proposed system. In addition, more case studies should be done to test the system in order to establish its capability and reliability. A good suggestion is by using different type of neural networks and learning algorithms could be employed.

\section{ACKNOWLEDGEMENTS}

This research work is supported by the (RAGS/1/2014/TK03/UPNM/2) research grant.

\section{REFERENCES}

[1] Haykin S. Neural network: A comprehensive foundation.New Jersey: Prentice Hall, 1994

[2] Suparta W, Adnan J,Ali M A M. The impacts of GPS PWV during heavy thunderstorms over Kuala Terengganu, Malaysia.In IEEE International in Green and Ubiquitous Technology, 2012, pp. 50-54

[3] Suparta W, Adnan J, Ali M A M. Direct impact monitoring of lightning activity on the GPS signals propagation. Australian Journal of Basic and Applied Sciences, 2011, 
5(9):604-609

[4] Suparta W, Adnan J, Ali M A M. Detection of lightning activity using GPS PWV measurements.In IEEE International Conference on Space Science and Communication, 2011, pp. $115-120$

[5] Suparta W, Adnan J, Ali M A M. Monitoring the association between GPS PWV and lightning activity during the 2009 Winter Monsoon over Bangi Malaysia.In International Conference on Environment Science and Engineering, 2011, pp. 101-106

[6] Suparta W, Adnan J, Ali M A M. Investigation of GPS PWV variation during lightning activity due to topographic effects.In IEEE International Conference on Space Science and Communication, 2013, pp. 115-119

[7] Isa N A, Hashim F R, Mei F W, Ramli D A, Omar W M, Zamli K Z. Predicting quality of river's water based on algae composition using artificial neural network.In 4th IEEE International Conference on Industrial Informatics, 2006, pp. 1340-1345

[8] Ramli D A, Saleh J M, Hashim F R, Isa N A. Multilayered Perceptron (MLP) network trained by recursive least squares algorithm. In 1st IEEE International Conference on Computers, Communications, and Signal Processing with Special Track on Biomedical Engineering, 2005, pp. 288-291

[15] Hush D R, Home B G. Progress in supervised neural networks. IEEE Signal Processing Magazine, 1993, 10(1):8-39

[16] El-Falahi, AA, Marti, R A, Lasdon L B. Path relinking and GRG for artificial neural networks. European Journal of Operational Research, 2006, 169(2):508-519

[17]Jayavanth S, Singh M. Artificial neural network analysis of malaria severity through aggregation and deformability parameters of erythrocytes. Clinical Hemorheology and Microcirculation, 2003, 29(3-4):457-468

[18]Battiti R. First and second-order method for learning between steepest descent and Newton's method. Neural Computation, 1992, 4(2):141-166

[19] Wang D, Lu W Z. Forecasting of ozone level in the time series using MLP model with a novel hybrid training algorithm. Atmosphere Environment, 2006, 40(5):913-924

[20]MathWorks. Neural network toolbox. Massachusetts:MathWorks Inc., 2017 
[21]KolkwitzR, Marsson M. Ecology of herbal Saprobias. Ber. d. Deut. Bot. Company, 1908, 26(2):505-519

[22] Mat Isa N A, Mashor M Y, Othman N H. Classification of cervical cancer using HMLP network with confident level analysis. International Journal of Computer, the Internet and Management. 2003, 11(1):17-29

[23]MitraP, Mitra S, Pal S K. Stagingof cervical cancer with soft computing. IEEE Transaction on Biomedical Engineering. 2000, 47(7):934-940

[24] Yassin I M, Jailani R, Ali M, Baharom R, Hassan A, Rizman Z I. Comparison between cascade forward and multi-layer perceptron neural networks for NARX functional electrical stimulation (FES)-based muscle model. International Journal on Advanced Science, Engineering and Information Technology, 2017, 7(1):215-221

\section{How to cite this article:}

Hashim F R, Nik Daud N G, Ahmad K A, Adnan J, Rizman Z I. Prediction of rainfall based on weather parameter using artificial neural network. J. Fundam. Appl. Sci., 2017, 9(3S), 493-502 